\title{
Link Budget Analysis and Modeling of Short-Range UWB Channels
}

\author{
Zoubir Irahhauten, Student Member, IEEE, Javier Dacuña, Student Member, IEEE, \\ Gerard J. M. Janssen, Member, IEEE, Homayoun Nikookar, Senior Member, IEEE, \\ Alexander G. Yarovoy, Senior Member, IEEE, and Leo P. Ligthart, Fellow, IEEE
}

\begin{abstract}
Ultrawideband (UWB) technology is an attractive alternative for short-range applications, e.g., wireless personal area networks. In these applications, transmit and receive antennas are very close to each other and the far-field condition assumed in most of the link budget models may not be satisfied. Under near-field conditions, variations in the link budget and pulse shape compared to the far-field can be observed. In this work, a novel line-of-sight UWB link budget model for very short distances is proposed and validated with measurements using different types of antennas. The proposed model, which takes into account all main near-field effects (i.e., phase error, antenna mismatch and reradiation between antennas) and includes frequency, antenna size and orientation as parameters, shows a good agreement with the simulations and the measurements. This model has also been applied to dispersive antennas (e.g., notch-antennas), and can be used in the design and analysis of short-range UWB communication systems.
\end{abstract}

Index Terms - Link budget, near-field measurements, ultrawideband (UWB) antenna, UWB propagation channel.

\section{INTRODUCTION}

$\mathbf{U}$ LTRAWIDEBAND (UWB) is a technology which was recently introduced for wireless communication systems, especially for short-range indoor applications. The Federal Communications Commission (FCC) has recognized the significance of UWB and initiated the regulatory review process of the technology. As a result, the UWB technology has been authorized for commercial use with different applications, different operating frequency bands as well as corresponding transmitted power spectral densities (PSD) [1].

The restrictions in the maximum PSD imposed by the FCC make this technology suitable for short-range networks, e.g., wireless personal area networks (WPAN). However, in these applications, transmit and receive antennas can be very close to each other (within the near-field region). Under near-field conditions, the common link budget models are not valid because

Manuscript received July 27, 2007; revised November 8, 2007. Published August 6, 2008 (projected). This work was supported by the Free-band Air-Link project (DTC. 5961).

Z. Irahhauten, G. J. M. Janssen, H. Nikookar, A. G. Yarovoy, and L. P. Ligthart are with the International Research Center for Telecommunications and Radar (IRCTR), Faculty of Electrical Engineering, Mathematics and Computer Science (EEMCS), Delft University of Technology, 2628 CD Delft, The Netherlands (e-mail: Z.Irahhauten@ewi.tudelft.nl).

J. Dacuña was with the International Research Center for Telecommunications and Radar (IRCTR), Faculty of Electrical Engineering, Mathematics and Computer Science (EEMCS), Delft University of Technology, 2628 CD Delft, The Netherlands. He is now with the Technical University of Catalonia (UPC), 1-3 08034 Barcelona, Spain.

Color versions of one or more of the figures in this paper are available online at http://ieeexplore.ieee.org.

Digital Object Identifier 10.1109/TAP.2008.927542 the behavior of the antennas is quite different from the behavior in the far-field. In the far-field, plane wave propagation and a constant ratio between the electric and magnetic field given by the wave impedance, is assumed. However, in the near-field region different effects such as reactive fields, phase errors, and even reradiation between the antennas may become important.

The design and implementation of short-range UWB communication systems need accurate channel models in order to optimize the transmit power and estimate the coverage. Several UWB measurement results for WPAN applications are reported in the literature [2]-[12]. In [3], the human body effect on UWB signal propagation is investigated, but only for the receive antenna near the body and the transmit antenna in the far-field. In [4], UWB channel measurements for a body area network (BAN) are presented where transmit and receive antenna are placed directly on the body, but only the $3-6 \mathrm{GHz}$ band was measured. Reference [6] shows the results of a set of time domain UWB measurements for very short distances. In spite of plenty of UWB measurement efforts for WPAN, the analysis of near-field effects and the link budget model has not received much attention.

In this paper, a novel UWB link budget model for short-range applications is proposed and validated with measurements and simulations. The near-field effects and its consequences on the UWB link budget are analyzed and its behavior is modeled. It turns out that known link budget models for the far-field can be applied, but with some modifications. The proposed model predicts the loss due to the near-field effects for short-range line-of-sight (LOS) UWB channels without any multipath effects. The structure of the paper is as follows. In Section II, the near-field effects are analyzed. The link budget model is proposed and explained in Section III. Section IV presents measurement results and verification of the proposed model. Concluding remarks are provided in Section V.

\section{NeAR-Field EFFECtS ANALysis Over a LARGE FREQUENCY BAND}

Different near-field criteria have been established based on different effects that can appear in this region [13]. In this section, these effects among others are analyzed with respect to the received power over a large frequency band.

\section{A. Phase Error}

The near-field criterion associated to phase error is restricted to the fact that an incident spherical wave differs from a plane wave in a given fraction of the wavelength $\lambda$. When transmit and 


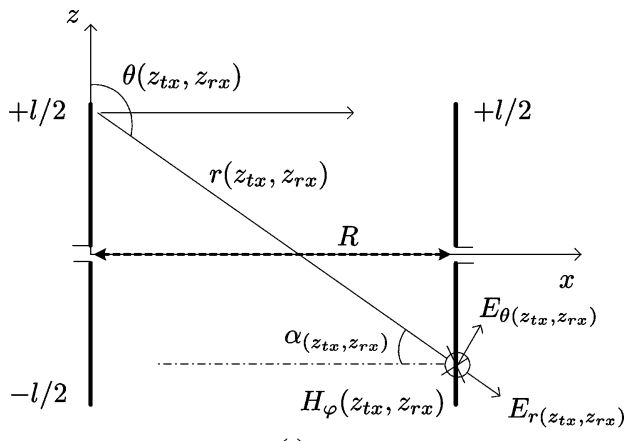

(a)

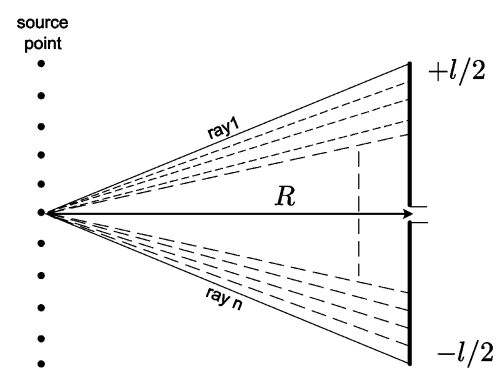

(b)

Fig. 1. Phase error between two dipoles.

receive antennas are close to each other, the rays of the spherical wave produced by the transmit antenna reach different parts of the receive antenna aperture with different phases. This affects the received power because the rays do not sum coherently at the receive antenna. The phase of each ray is a function of the distance between the antennas, the frequency and the antenna dimension. This effect causes variations in the received power as function of distance between the antennas and frequency and may cause distortion in the received signal. In the following, the near-field effect associated to the phase error is analyzed. Without loss of generality and for simplicity of the analysis, a dipole antenna with full matching is assumed. We analyze the case of two "thin" dipoles (i.e., transmit and receive) as depicted in Fig. 1(a). The voltage $V_{r x}$ at the terminals of the receive dipole can be expressed as [13]

$$
V_{r x} \propto \frac{1}{I_{r x_{i}}} \int_{-l / 2}^{+l / 2} E_{r z}\left(z_{r x}\right) I_{r x}\left(z_{r x}\right) d z_{r x}
$$

where $I_{r x_{i}}$ is the current at the feed point of the receive antenna, $I_{r x}$ is the current illumination of the receive dipole, $z_{r x}$ is the position along the receive dipole and $l$ is the length of the dipole. The electric field along the receive dipole is given by:

$$
\begin{aligned}
E_{r z}\left(z_{r x}\right)=[ & \int_{-l / 2}^{+l / 2} E_{\theta}\left(z_{t x}, z_{r x}\right) \cos \left(\alpha\left(z_{t x}, z_{r x}\right)\right) d z_{t x} \\
& +\int_{-l / 2}^{+l / 2} E_{r}\left(z_{t x}, z_{r x}\right) \\
& \left.\times \cos \left(\frac{\pi}{2}-\alpha\left(z_{t x}, z_{r x}\right)\right) d z_{t x}\right] \hat{z} .
\end{aligned}
$$

Using the equations of the infinitesimal dipole as given in [13]

$$
E_{r}=\eta \frac{I_{0} l \cos \theta}{2 \pi r^{2}}\left[1+\frac{1}{j k r}\right] e^{-j k r}
$$
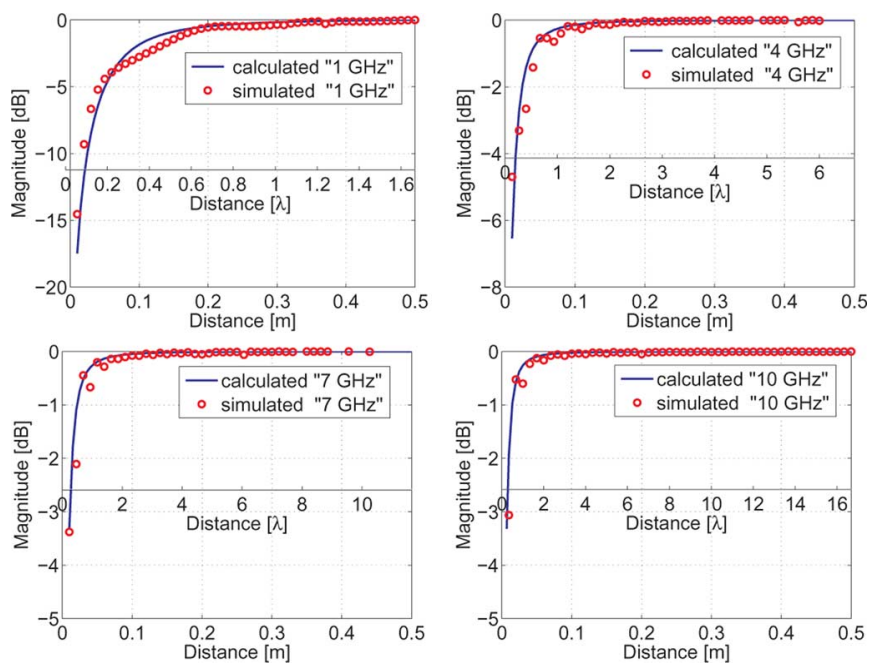

Fig. 2. Comparison of calculated and simulated received power as a function of distance for different dipoles.

$$
E_{\theta}=j \eta \frac{k I_{0} l \sin \theta}{4 \pi r}\left[1+\frac{1}{j k r}-\frac{1}{(k r)^{2}}\right] e^{-j k r} .
$$

The voltage in the receive antenna can be expressed as

$$
V_{r x} \propto \frac{1}{I_{r x_{i}}} \int_{-l / 2}^{l / 2}\left[E_{1}+E_{2}\right] I_{r x}\left(z_{r x}\right) d z_{r x}
$$

where

$$
\begin{aligned}
E_{1}=\int_{-l / 2}^{l / 2}[ & j \eta \frac{k I\left(z_{t x}\right) l \sin \theta\left(z_{t x}, z_{r x}\right)}{4 \pi r\left(z_{t x}, z_{r x}\right)} \\
& \times\left[1+\frac{1}{j k r\left(z_{t x}, z_{r x}\right)}-\frac{1}{\left(k r\left(z_{t x}, z_{r x}\right)\right)^{2}}\right] \\
& \left.\times e^{-j k r\left(z_{t x}, z_{r x}\right)} \cos \left(\alpha\left(z_{t x}, z_{r x}\right)\right)\right] d z_{t x} \\
E_{2}=\int_{-l / 2}^{l / 2}[ & \eta \frac{I\left(z_{t x}\right) l \cos \theta\left(z_{t x}, z_{r x}\right)}{2 \pi r\left(z_{t x}, z_{r x}\right)^{2}}\left[1+\frac{1}{j k r\left(z_{t x}, z_{r x}\right)}\right] \\
& \left.\times e^{-j k r\left(z_{t x}, z_{r x}\right)} \cos \left(\frac{\pi}{2}-\alpha\left(z_{t x}, z_{r x}\right)\right)\right] d z_{t x}
\end{aligned}
$$

where $k$ is the wave number $(2 \pi / \lambda), I_{0}$ is the excitation current, $\eta$ is the free space impedance $(120 \pi \Omega), \theta$ is the elevation angle with respect to the axis of the dipole assumed along the zenith, $\phi$ is the azimuth angle with respect to the $x$-axis in a rectangular coordinate system with its $z$-axis along the zenith, and $r$ is the distance from the dipole. To verify expression (5), simulations were done using the IE3D Electromagnetic simulator. ${ }^{1}$ To this end, different dipoles associated to different frequencies (i.e., different lengths) have been simulated. Fig. 2 compares the calculated and simulated (normalized) received power as a function of distance between two dipoles (the x-axis in the middle of the figures shows the distance normalized to the wavelength $\lambda)$. The received power $\left(20 \log _{10}\left(V_{r x}\right)\right)$ is normalized to the distance assuming a distance dependence of $1 / R^{2}$. From this figure it can be observed that the received power at very short

\footnotetext{
${ }^{1}$ More details about the IE3D simulator is available at www.zeland.com
} 




(a)

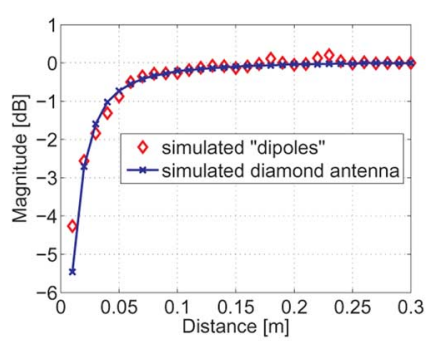

(b)
Fig. 3. Normalized received power, averaged over the frequency band 3 to $10 \mathrm{GHz}$, as a function of the distance. (a) Calculated versus combined dipoles. (b) Combined dipoles versus UWB diamond antenna.

distances is less than expected for the far-field region of the antenna. The main reason is that different rays picked up by the antenna are not in-phase, and therefore the total received power is less. Another reason is that when a source point is very close to the dipole at distance $R$, the rays of the received spherical wave have traveled a distance larger or equal to $R$ as can be seen from Fig. 1(b). It can also be seen that the calculations match well to the simulations and they experience the same (exponential) behavior. Moreover, the loss is higher for larger antennas, which agrees with the phase error criterion. According to the obtained results, the received power experiences the same behavior with respect to frequency and hence the same behavior can be expected when the power is averaged over a large frequency band. Therefore, the simulation results of different dipoles at different center frequencies are combined. Fig. 3(a) shows the calculated and simulated normalized power, averaged over the frequency band 3-10 GHz (i.e., 8 dipoles of 3 to $10 \mathrm{GHz}$ with a step of $1 \mathrm{GHz}$ ), as a function of the distance between two dipoles. Again the results match very well. However, a dipole antenna is known as a narrowband antenna. To validate whether this approach is correct, additional simulations with wideband antennas have been done. To this end, a diamond antenna ${ }^{2}$ has been simulated between 3 and $10 \mathrm{GHz}$ and the results were compared to those of combined dipoles in Fig. 3(b), from which identical behavior is observed.

\section{B. Antenna Mismatch}

The electric and magnetic fields for distances within the nearfield region have a quite different behavior. They consist of radiating components and reactive or storage components. For radiating fields, the source is not affected by the observer. Once the radiating field leaves the antenna, it is gone forever, and the source is not affected if the energy is absorbed or not. For reactive or stored fields the effect is different. Any time that an observer extracts or diverts part of the reactive field energy, it will cause a reaction in the source circuit which might change the input impedance of the antenna [14]. Fig. 4(a) shows the progression of the different terms in (4) as a function of the distance. In this figure, it can be seen which field predominates in the nearand far-field. All the terms have the same value at $\lambda / 2 \pi$. For distances below $r=\lambda / 2 \pi$ (point of intersection) the reactive fields predominate. The coupling of the reactive static field to the receive antenna can be modeled as an electric and/or magnetic

\footnotetext{
${ }^{2} \mathrm{~A}$ diamond antenna is a planar antenna formed by two isosceles triangles. The width and height of the triangles is about $\lambda / 4$ of the center frequency.
}

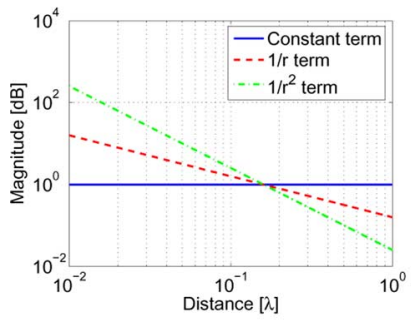

(a) Radiating and reactive fileds

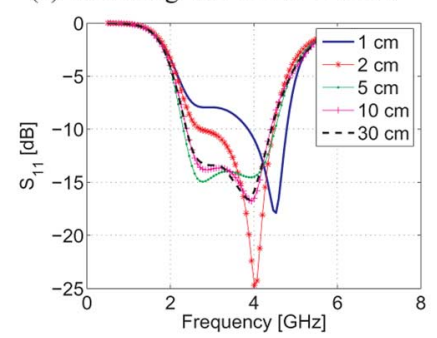

(c) $3 \times 3 \mathrm{~cm}$ diamond antenna

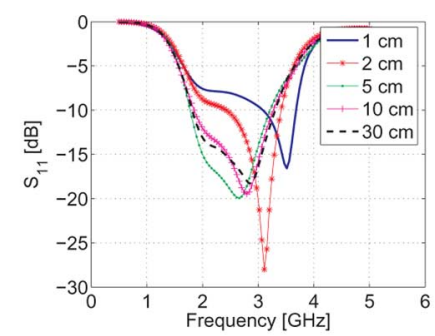

(b) $4 \times 4 \mathrm{~cm}$ diamond antenna



(d) $2 \times 2 \mathrm{~cm}$ diamond antenna.
Fig. 4. Variations of the reflection coefficient as a function of frequency for different distances between antennas.

coupling and it will affect the transmit antenna by changing its input impedance. This coupling will vary with frequency. The changes in the input impedance of the transmit antenna may cause a mismatch for one or several frequencies, and a distortion in the frequency response of the antennas. To analyze this effect, simulations have been done using a diamond antenna with a second identical antenna placed at different distances. Fig. 4(b)-(d) shows the antenna reflection coefficient $S_{11}$ as a function of frequency for different separation distances using diamond antennas. Different frequency bands are simulated according to different "diamond" antenna sizes (i.e., $2 \times 2 \mathrm{~cm}$ and $4 \times 4 \mathrm{~cm}$ for one flair). From these figures it can be seen that for distances of $2 \mathrm{~cm}$ and larger the variations in $S_{11}$ are not very big because the antenna is still matched. For distances of $1 \mathrm{~cm}$ (or less) a small antenna mismatch occurs. It can also be concluded that the mismatching is more important for large antennas than for small antennas.

\section{Reradiation Between Antennas}

The voltage induced in a receive antenna due to the electromagnetic field generated by a transmit antenna produces a current through both the antenna impedance and the load impedance. Part of the power picked up by the antenna will be absorbed by the load impedance and a part of the power will be dissipated in the antenna impedance. This might produce a reradiation from the receive antenna that can be picked up by the transmit antenna and reradiated again producing interference in the receive antenna. The amount of power reradiated by the receive antenna depends on the antenna mismatch and the physical antenna structure. For a given distance, the reradiation between the antennas may add constructively or destructively depending on the frequency and the phase shift introduced by both antennas. This effect causes a variation of the received power as a function of frequency. This means that the channel frequency response may change for different distances, not only in level, but also the shape. The previous simulation results are used to investigate the received pulse waveform for different 



(a) Antenna size "2 cm"


(b) Antenna size "3 cm"
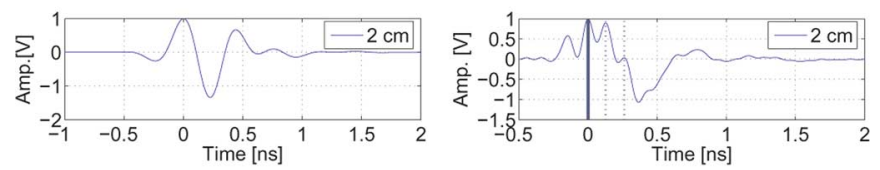

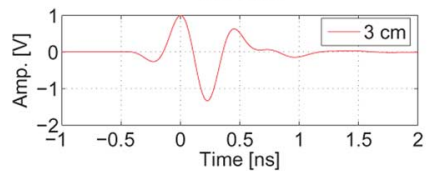

(c) Antenna size "4 cm"

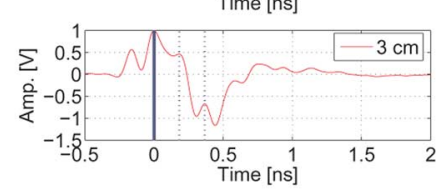

(d) Antenna size "6 cm"
Fig. 5. Received pulse waveform at 2 and $3 \mathrm{~cm}$ distance for different diamond antenna sizes.

distances between two diamond antennas. Fig. 5(a)-(c) shows the received pulse waveform for diamond antennas with sizes of 2,3 , and $4 \mathrm{~cm}$, respectively. From these figures, the received pulse waveform at $2 \mathrm{~cm}$ and $3 \mathrm{~cm}$ distance is almost the same and hence the reradiation between the antennas is negligible. Clearly, for a smaller antenna the effect is smaller, due to the dependence between the surface of the antenna and the structural mode of the scattering cross section. Therefore, to highlight this effect, an additional simulation of a larger $6 \mathrm{~cm}$ diamond antenna was done. The corresponding results are shown in Fig. 5(d). From this figure, the reradiation between antennas is clearly seen. The vertical dashed lines mark an interval equivalent to two times the distance between the antennas in order to have a reference of the "repetition" of the pulse due to antenna reradiation.

\section{PROPosed Link Budget Model}

Different effects such as antenna mismatch, reradiation between antennas and phase error can appear when transmit and receive antennas are close to each other. As UWB is mainly introduced for indoor communications and the frequency band proposed by FCC for such environment is 3.1-10.6 $\mathrm{GHz}$, the antenna mismatch is restricted to distances smaller than $c / 2 \pi f_{\min } \approx 1.5 \mathrm{~cm}$. Thus, this effect will be negligible for most of the applications. Reradiation between antennas mainly appear for large antennas which are not often used for short-range applications. As a result, the link budget in the near-field will be primarily affected by the phase error which is dependent on the considered bandwidth. When the received power is integrated over the large UWB bandwidth, it tends to be smaller than the expected received power using Friis law, as can be seen from Fig. 3. The Friis equation is given by

$$
P_{r}(f)=P_{t}(f) G_{t}(f) G_{r}(f)\left(\frac{\lambda}{4 \pi r}\right)^{2}
$$

where $\lambda=c / f$ and $P_{t}, P_{r}, G_{t}$ and $G_{r}$ are the transmit and receive powers, and transmit and receive antenna gains, respectively. The phase error becomes important when the physical dimensions of the antennas are large compared to the distance between the antennas, i.e., for distances $r<2 D^{2} / \lambda=r_{f f}[13]$ where $D$ is the largest dimension of the antenna and $r_{f f}$ is the far-field limit distance. The operating frequency also plays an important role because at higher frequencies the phase errors will be larger for the same antenna dimensions, and the behavior will be different for different frequencies, which implies that it can result in signal distortion. Thus, these parameters should be taken into account in the model of the channel under near-field conditions. Channel modeling of near-field effects based on (5) is complicated. In this paper, we propose a simple model for the link budget in the near-field. The novel proposed model introduces an extra loss factor to the known far-field free space equation, caused by the near-field effects. In this model, the total loss is expressed as

$$
L_{\text {total }}(r, f)=\frac{P_{t}(f)}{P_{r}(f)}=L_{f f}(r, f) \gamma_{n f}(r, f)
$$

where

$$
\gamma_{n f}(r, f)=1-e^{-r / \delta_{D}\left(f, A_{s}, \theta\right)}
$$

$L_{\text {total }}$ is the total loss of the channel, $L_{f f}$ is the channel loss in the far-field, $\gamma_{n f}$ is the correction factor due to near-field effects and $\delta_{D}$ is the distance decay constant which depends on the maximum size of the antenna $A_{s}$, its orientation $\theta$ and the frequency $f$. According to (10), the near-field correction factor $\gamma_{n f} \approx 1$ for the far-field case $r \gg \delta_{D}$. The proposed model is validated with measurements for different kinds of antennas.

\section{MEAsurements, Simulations AND VERIFICATION}

\section{A. Measurement Setup}

In order to validate the proposed model, a set of measurements has been performed at different distances using a time domain technique as shown in Fig. 6(a). The generator fires a Gaussian-like pulse with a time duration of $35 \mathrm{ps}$. The sampling oscilloscope controls the sampling unit and the pulse generator with trigger pulses each $100 \mathrm{~ns}$ and has an operational bandwidth up to $26 \mathrm{GHz}$. An acquisition time window of $10 \mathrm{~ns}$ is used with 4096 points which means that the received signal is sampled at a rate of 1 sample per 2 ps. The antennas are situated on Styrofoam boards in order to keep them more than one meter from the ground. Also there were no objects at less than one meter from the antennas. In this way, the reflections of the walls and/or objects can be removed using an appropriate time window. In addition to time domain measurements, a set of measurements based on the frequency domain technique has been performed in an anechoic chamber [see Fig. 6(b)]. To this end, a network analyzer "Agilent-E8364B" is used and a bandwidth of $12 \mathrm{GHz}$ is measured from 0.01 to $12.01 \mathrm{GHz}$ with a step size of $7.5 \mathrm{MHz}$. For both setups, two identical transmit and receive antennas were used. One antenna was kept fixed while the other one was moved on a straight line covering a distance from 


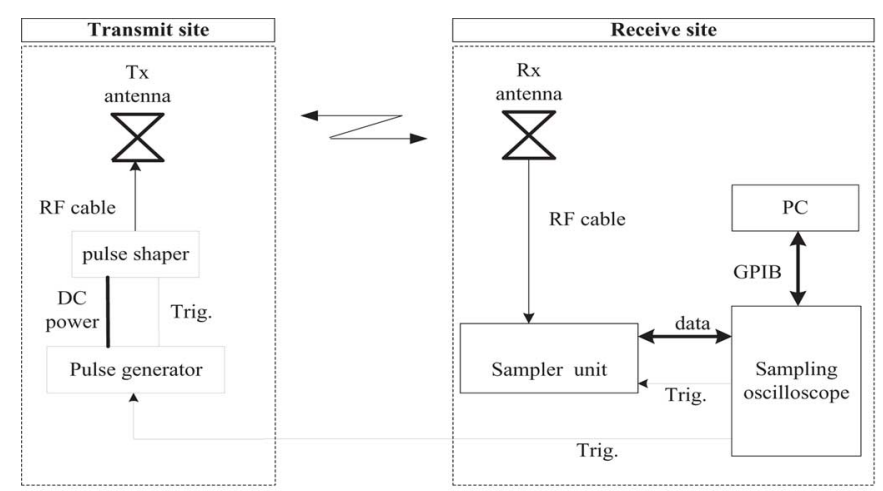

(a) Time domain setup



(b) Frequency domain setup

Fig. 6. The setups used for the measurements.

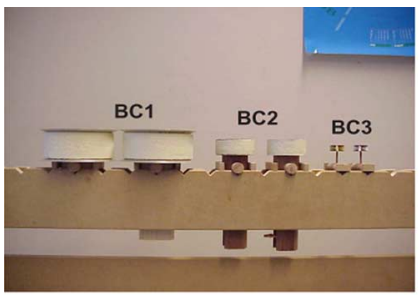

(a) Bi-conical antennas

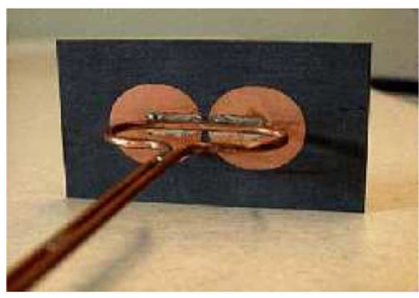

(c) Butterfly antenna

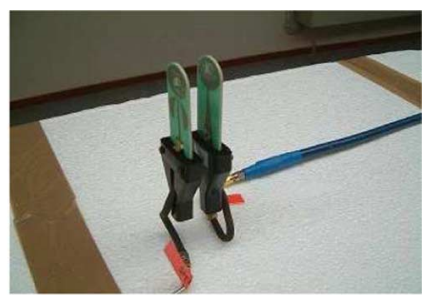

(b) Time Domain antenna

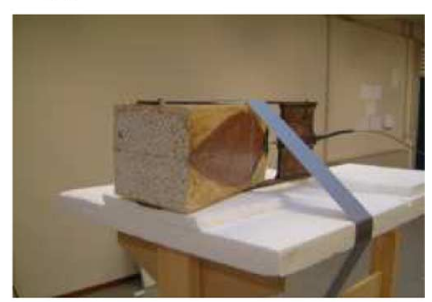

(d) Dielectric Wedge antenna
Fig. 7. Different antennas used for the measurements.

$0.5 \mathrm{~cm}$ to $1 \mathrm{~m}$. In total six pairs $(6 \times 2)$ of different antennas were used (see Fig. 7):

- three pairs are bi-conical antennas with different sizes and referred to as $\mathrm{BC} 1, \mathrm{BC} 2$ and $\mathrm{BC} 3$, with their dimensions, [diameter, height] in centimeters: $[16,6.5],[7.5,3.2]$ and [2.6, 0.8], respectively;

- one pair consists of planar elliptical UWB time domain "Schantz" antennas (2-8 GHz) [15];

- one pair consists of planar UWB butterfly antennas (2-9 GHz) [16];

- one pair consists of dielectric wedge antennas $(0.5-6 \mathrm{GHz})$ [17].
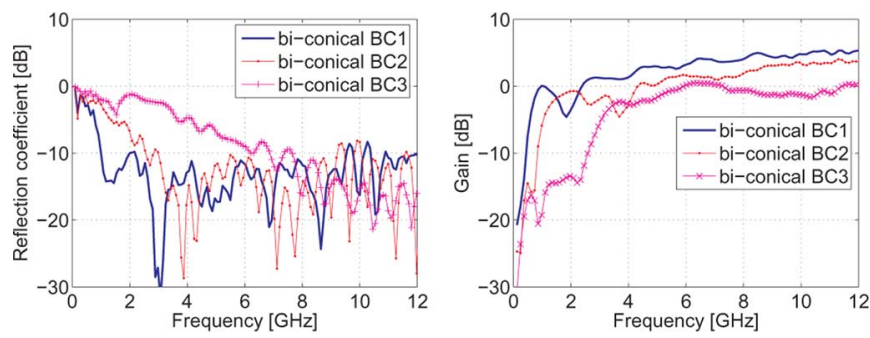

Fig. 8. The frequency characteristics of the bi-conical antennas used for the measurements.

The reflection coefficient and the gain of the bi-conical antennas are shown in Fig. 8. More details about other antennas can be found in [15], [16] and [17], respectively. In total, 1400 measured profiles have been gathered.

\section{B. Data Processing Method}

First the received time domain signal is filtered over the entire antenna bandwidth using a digital butterworth filter. In this way the out-of-band noise is suppressed. Then, a raised cosine window is implemented to remove undesired signals and also to reduce the leakage problem when transforming the signal to the frequency domain. The received signal is normalized to the free space equation as follows. The measured voltage in the far-field can be expressed using the Friis equation as

$$
V_{r}^{f f}(f)=V_{g}(f) \sqrt{G_{t}(f) G_{r}(f) G_{s y s}(f)}\left(\frac{\lambda}{4 \pi r_{f f}}\right)
$$

where $V_{g}(f)$ is the generator output, $G_{t}(f)$ and $G_{r}(f)$ are transmit and receive antenna gains, respectively, $r_{f f}$ is a farfield distance and $G_{s y s}(f)$ is the transfer function of the measurement system which includes the cables, attenuators, etc. The measured voltage in the near-field can be expressed now as

$$
\begin{aligned}
V_{r}^{n f}(f)=\left[V_{g}(f) \sqrt{G_{t}(f) G_{r}(f) G_{s y s}(f)}\left(\frac{\lambda}{4 \pi r_{n f}}\right)\right] \\
\\
\times \gamma_{n f}(r, f)
\end{aligned}
$$

where $r_{n f}$ is a near-field distance. Using (11) and (12), we get

$$
\gamma_{n f}(r, f)=\left[\frac{r_{n f}}{r_{f f}} \frac{1}{V_{r}^{f f}(f)}\right] V_{r}^{n f}(f) .
$$

From this equation it can be seen that the modeling results do not depend on the signal used to excite the channel which makes the model easy to use in practice.

\section{Measurement and Modeling Results}

The total normalized received power is integrated over the entire antenna bandwidth (e.g., 3-10 GHz) in order to obtain the received power as a function of the distance between the antennas. From the measurements, the distance decay constant $\delta_{D}$ is estimated using the least squares method and the results of the model are plotted and compared to the measured results. Fig. 9 shows the measurement results of the normalized received power as a function of the distance for different antennas. For the bi-conical antennas the measured distance is determined as 

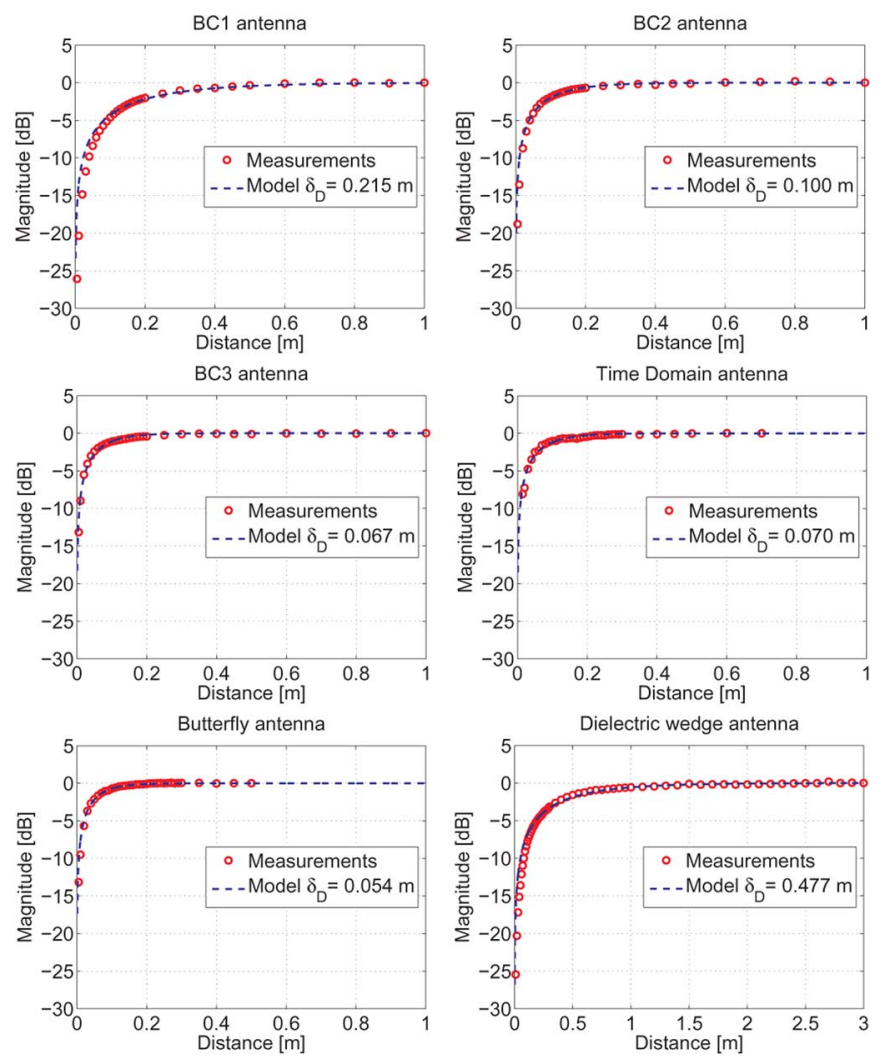

Fig. 9. Normalized channel loss as a function of distance for different types of antennas.
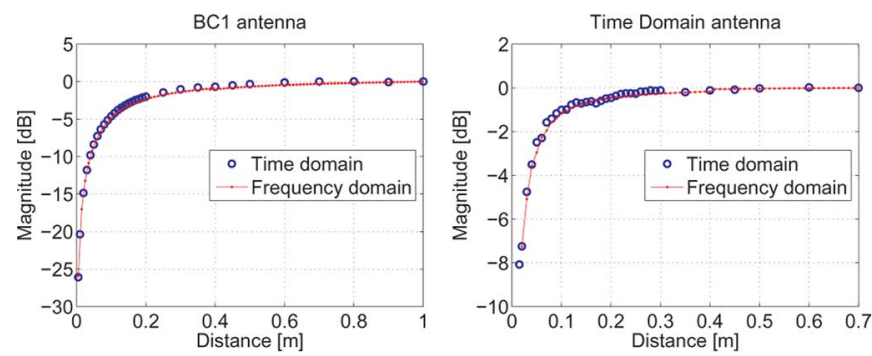

Fig. 10. Comparison of the measurement results using time and frequency domain setups.

the distance between the edges of transmit and receive antennas. We consider the edge of the antenna as the reference point because that is the point where most of the energy radiates. As the magnitude of the received power is normalized to the far-field, the loss due the near-field effects can be seen as the difference between the curves and the horizontal $0 \mathrm{~dB}$ line. From this figure it can be observed that for all used antennas the extra loss due to near-field conditions decreases with the separation distance until $r_{f f}$, and it has an exponential behavior. The measurements and the proposed model match well for all antennas. Additionally, to check the validity of the model, the time domain results are compared to those obtained with the frequency domain technique as shown in Fig. 10. It is clear that the modeling results are the same for both techniques.

Moreover, the measured signal experiences all near-field effects discussed in Section II. In the following we try to check the impact of those effects among others by means of measurements rather than by simulations. Fig. 11 shows the measured results of
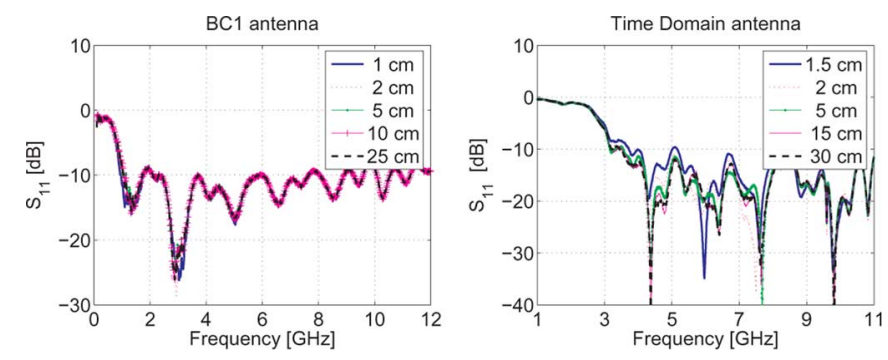

Fig. 11. The measured reflection coefficient as a function of frequency for different separation distances.



(a) Bi-conical antenna $\mathrm{BC} 1$

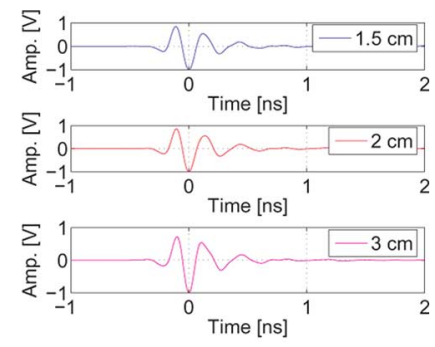

(b) Time Domain antenna
Fig. 12. The measured pulse waveform at different separation distances.

the reflection coefficient for the bi-conical "BC1" antenna and the Time Domain antenna. It can be seen that for the $\mathrm{BC} 1$ antenna the $S_{11}$ parameter does not change with the distance. The same effect is observed for the Time Domain antenna. Only a very small variation is seen at a distance of $1.5 \mathrm{~cm}$, however, the $S_{11}$ is below $-10 \mathrm{~dB}$ which means that the antenna is still matched. To investigate the reradiation between antennas, the measured signals at different "small" distances are compared in Fig. 12. From this figure it is clear that antenna reradiation does not occur.

\section{Model Parameters}

1) Antenna Size: According to the results in Fig. 9, it can be concluded that for a fixed distance the losses are larger for larger antennas and this agrees with the phase error definition. The distance for which the extra loss is $3 \mathrm{~dB}$ is about $15,7.1$, and $4 \mathrm{~cm}$ for the $\mathrm{BC} 1, \mathrm{BC} 2$, and $\mathrm{BC} 3$ antennas, respectively. These values are comparable with the maximum size of each antenna, that is $17.3 \mathrm{~cm}, 8.2 \mathrm{~cm}$ and $3.5 \mathrm{~cm}$, respectively. Fig. 13(a) shows the distance decay constant $\delta_{D}$ as a function of the maximum size for the bi-conical antennas $\mathrm{BC} 1, \mathrm{BC} 2$, and $\mathrm{BC} 3$. Although limited data was available, it can generally be observed that the behavior of $\delta_{D}$ tends to increase with the maximum antenna size $A_{s}$. The same behavior is observed for the simulated diamond antennas. Fig. 13(b) shows the parameter $\delta_{D}$ versus $A_{s}$ for all used antennas. The solid line represents a best fit model for the variation of $\delta_{D}$ with $A_{s}$. Using this model and the size of any other selected antenna, a-priori information about the parameter $\delta_{D}$ can be obtained.

2) Frequency Dependency: As stated before, the coupling between antennas as well as the phase error will vary with the operating frequency. Therefore, it is important to check the frequency dependency of the proposed model. To this end, the large bandwidth is divided into small frequency bands (i.e., chunks), and the model parameter $\delta_{D}$ is estimated for each chunk (e.g., $100 \mathrm{MHz}$ ). 




(a) Bi-conical antennas

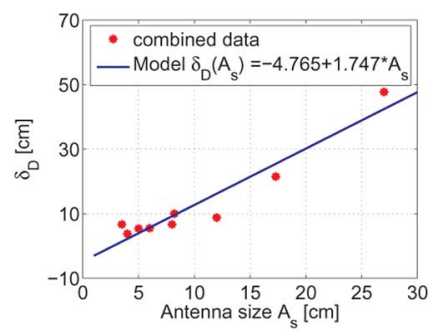

(b) Combined data of all antennas
Fig. 13. Distance decay constant versus maximum antenna size.

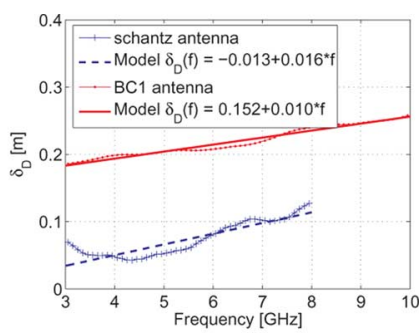

(a) Measured BC1 and TD antennas



(b) Simulated diamond antennas
Fig. 14. Distance decay constant as a function of frequency.



(a) The reflection coefficient

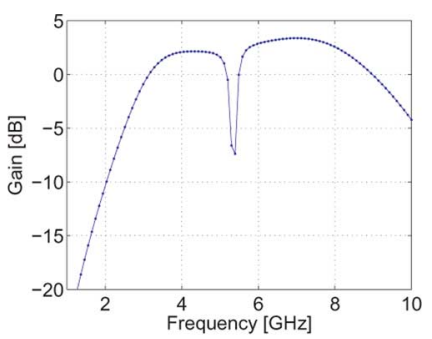

(b) The gain
Fig. 15. Frequency characteristics of the UWB notch antenna.

Fig. 14 gives the measurement and simulation results of $\delta_{D}$ as a function of frequency which show that $\delta_{D}$ increases slightly with frequency and the slope is almost the same for all cases. Based on these results, the parameter $\delta_{D}$ can be modeled as:

$$
\delta_{D}(f)=a+b f
$$

where the constant $a$ depends on the size of the antenna and the slope $b$ depends on the geometry of the antenna.

3) The Effect of Dispersive Antennas: One of the major problems in UWB transmission is the narrow-band interference (i.e., from WLAN). To suppress such interference different solutions have been proposed. One classical solution is using a notch filter which can be designed into the antenna. When an antenna has a notch it will become time dispersive. The proposed model is checked for such kind of dispersive antennas by means of simulations using a diamond antenna of almost $2 \mathrm{~cm}$ with a notch at $5.4 \mathrm{GHz}{ }^{3}$ The reflection coefficient $S_{11}$ and the gain $S_{21}$ of the antenna are shown in Fig. 15. Fig. 16(a) gives the simulation results of the normalized power as a function of distance for the antenna with and without the notch. The obtained results are almost the same and this is because the power is integrated over a large bandwidth and hence the notch is well compensated. However, we expect that when the power is integrated over a smaller

\footnotetext{
${ }^{3}$ Please note that the objective and emphasis of this part is applying the pro-
} posed model to a dispersive antenna rather than notch antenna design.

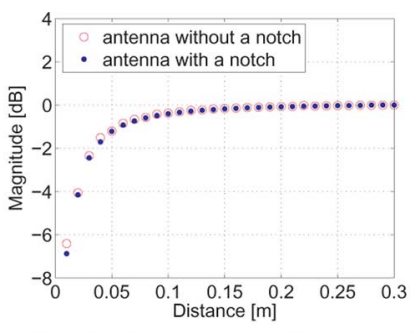

(a) Received power as a function of distance

Fig. 16. Comparison of diamond antenna with and without a notch.

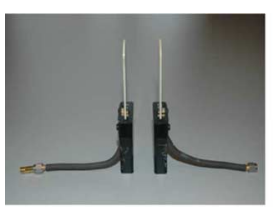

(a) 0 degree

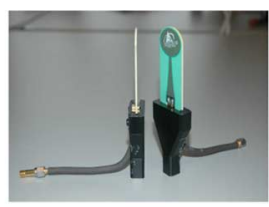

(b) 45 degrees



(c) 90 degrees
Fig. 17. Illustration of different orientation angles.

bandwidth, the notch will dramatically influence the model parameter. To check this, we evaluate the frequency dependency of $\delta_{D}$. Fig. 16(b) gives the simulation results of $\delta_{D}$ as a function of frequency for the antenna with and without a notch. From this figure it is clear that the deviation of $\delta_{D}$ from the model is large around the notch. Exactly at the notch-frequency, $\delta_{D}$ is equal to zero because the received power for all distances converge to that notch point and hence the difference between the received power is zero which means no difference between near and far-field. It should also be noted that at higher frequencies there is a deviation of $\delta_{D}$ between the two cases and this is because the antennas do not have exactly the same size.

4) Antenna Orientation: For near-field measurements the radiation pattern of an antenna is not formed. Parameters as gain, directivity, etc. have no meaning in this region because they are specifically defined assuming far-field conditions. However, since the phase error and the coupling depend on the position of the antenna the model is checked for different antenna orientations. For this reason, a set of measurements is performed keeping one antenna fix while changing the orientation of the other antenna from -90 to +90 degrees with a step of 5 degrees. This is repeated for all distances between transmit and receive antenna using the Time Domain antennas. Fig. 17 shows the position of the antennas for different orientation angles. Fig. 18 shows the measurement results of the normalized received power as a function of distance for different orientation angles. From this figure we can see that the proposed model is still valid for all orientation angles. Moreover, the loss at 0 degree is maximum. This can be explained by the fact that when the antenna is oriented at a certain angle, for example 90 degrees, a half of its aperture is situated more in the near-field region than the other half where its received power is higher and consequently the total power is higher.

\section{CONCLUSION}

In this work, a new LOS UWB link budget model for short-range applications under near-field antenna conditions 


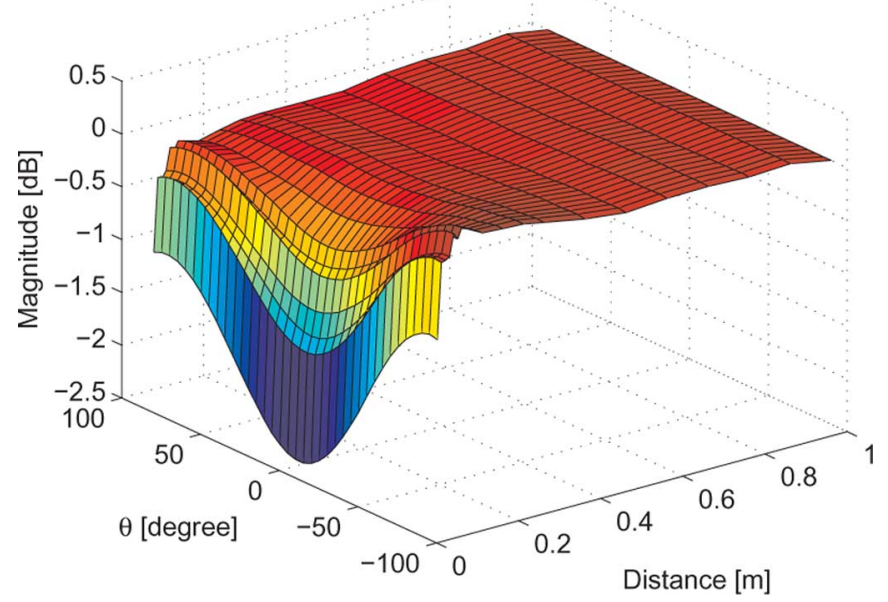

Fig. 18. Normalized received power versus distance for different orientation angles $\theta$.

is proposed and validated by means of simulations and measurements. The model introduces a near-field modification factor for the losses as predicted under far-field conditions. The proposed model takes into account all main effects due to the near-field conditions (i.e., phase error, antenna mismatch and reradiation between antennas) and includes frequency, antenna size and orientation dependencies. A set of time and frequency domain UWB measurements covering the frequency band from 3.1-10.6 GHz was conducted using different antenna types. The model shows a good agreement with respect to the measurements and simulations and does not depend on the signal used to excite the channel which makes it easy to use in practice. The obtained results show that the received signal for small antennas is mainly influenced by the phase error, however for large antennas operating at low frequencies antenna mismatch and reradiation between antennas may become important as well. The near-field effect shows a strong dependency on the physical size of the antenna; it is stronger for larger antennas than for smaller antennas. The distance decay constant slightly increases with the frequency and is linearly modeled. The obtained results can be used in the design and analysis of short-range UWB communication systems.

\section{ACKNOWLEDGMENT}

The authors would like to thank P. Aubry for his assistance in performing the measurements and A. Vorobyov for providing us with the butterfly antennas. The authors acknowledge the anonymous reviewers for their useful comments.

\section{REFERENCES}

[1] "Revision of part 15 the commission's rules regarding ultra-wideband transmission systems," in ET Docket. Washington, DC: Federal Communications Commission (FCC), 2002.

[2] Y. Suzuki and T. Kobayashi, "Ultra wideband signal propagation in desktop environments," in Proc. IEEE Conf. on Ultra Wideband Systems and Technologies, Nov. 2003, vol. 47, pp. 493-497.

[3] T. B. Welch, R. L. Musselman, B. A. Emessiene, P. D. Gift, D. K. Choudhury, D. N. Cassadine, and S. M. Yano, "The effects of the human body on UWB signal propagation in an indoor environment," IEEE J. Sel. Areas Commun., vol. 20, pp. 1778-1782, Dec. 2003.

[4] T. Zasowski, F. Althaus, M. Stager, A. Wittneben, and G. Troster, "UWB for noninvasive wireless body area networks: Channel measurements and results," in Proc. IEEE Conf. on Ultra Wideband Systems and Technologies, Nov. 2003, pp. 285-289.
[5] C.-C. Chong and S. K. Yong, "A generic statistical-based UWB channel model for high-rise apartments," IEEE Trans. Antennas Propag., vol. 53, no. 8, pp. 2389-2399, Aug. 2005.

[6] Z. Irahhauten, J. Dacuña, G. J. M. Jannsen, and H. Nikookar, "UWB channel measurements and results for wireless personal area networks applications," in Proc. European Conf. on Wireless Technology, Oct. 2005, pp. 209-212.

[7] S. S. M. Wong, F. C. M. Lau, and C. K. Tse, "Propagation characteristics of UWB radio in a high-rise apartment," in Proc. 8th Int. Conf. on Advanced Communication Technology (ICACT), Feb. 2006, vol. 2, pp. 914-918.

[8] K. Leechaikitjaroen, S. Promwong, P. Supanakoon, S. Chensirikul, and S. Kaewmechai, "Indoor measurement results of UWB impulse radio for shot-range wireless systems with RMS delay spread and path loss," in Proc. IEEE Int. Symp. on Communications and Information Technology (ISCIT), Oct. 2005, vol. 1, pp. 684-688.

[9] J. Choi, N.-G. Kang, Y.-S. Sung, and S.-C. Kim, "Empirical ultra wide band path loss model in office environments," in Proc. IEEE 63rd Vehicular Technology Conf. VTC-Spring, Oct. 2006, vol. 4, pp. 1956-1960.

[10] A. Fort, C. Desset, J. Ryckaert, P. D. Doncker, L. V. Biesen, and S Donnay, "An ultra-wideband body area propagation channel model from statistics to implementations," IEEE Trans. Microw. Theory Tech., vol. 4, no. 4, pp. 1820-1826, Jun. 2006.

[11] A. Molisch, "A comprehensive standardized model for ultrawideband propagation channels," IEEE Trans. Antennas Propag., vol. 54, no. 11, pp. 3151-3166, Aug. 2006.

[12] X. W. P. C. Richardson and W. Stark, "Modeling of ultra-wideband channels within vehicles," IEEE J. Sel. Areas Commun., vol. 24, no. 4, pp. 906-912, Apr. 2006

[13] C. A. Balanis, Antenna Theory: Analysis and Design. New York: Wiley, 1997.

[14] R. Schmitt, Electromagnetics Explained: A Handbook for Wireless/RF, EMC, and High-Speed Electronics, ser. EDN Series for Design Engineers. The Netherlands: Newnes, 2002.

[15] H. G. Schantz, "Bottom fed planar elliptical UWB antennas," in Proc IEEE Conf. on Ultra Wideband Systems and Technologies, Nov. 2003 pp. 219-223.

[16] A. V. Vorobyov, J. H. Zijderfeld, A. G. Yarovoy, and L. P. Ligthart, "Impact common mode currents on miniaturized UWB antenna performance," in Proc. IEEE European Conf. on Wireless Technology, Paris, France, Oct. 2005.

[17] A. G. Yarovoy, A. D. Schukin, I. V. Kaploun, and L. P. Ligthart, "The dielectric wedge antenna," IEEE Trans. Antennas Propag., vol. 50, pp. $1460-1472$, Oct. 2002.

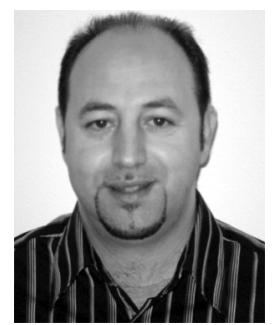

Zoubir Irahhauten (S'03) received the M.Sc. degree in electrical engineering from Delft University of Technology (TUDelft), Delft, The Netherlands, in 2002 , where he is currently working toward the Ph.D. degree.

$\mathrm{He}$ joined the International research Center for Telecommunications and Radar (IRCTR) as Researcher in the same year. In 2007, he joined the Circuits and Systems group of the Department of Electrical Engineering, Mathematics and Computer Science, TUDelft, where he is involved in under-water communication and positioning systems. His research interests include UWB channel modeling and measurements, UWB antenna design and positioning.

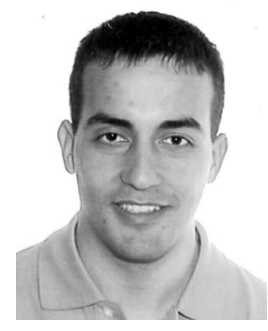

Javier Dacuña (S'08) received the B.Sc. and M.Sc. degrees in electrical engineering from the Technical University of Catalonia (UPC), Spain, in 2003 and 2005 , respectively, where he is currently working toward the Ph.D. degree.

He was an Exchange Student at the Wireless and Mobile Communications Group (WMC), Delft University of Technology (TUDelft), Delft, The Netherlands. His research interests include RFID, near-field communications, UWB communications and signal processing.

Mr. Dacuña received the First and Second National Prize to the Best Academic Record in Spain from the Education and Science Ministry (MEC), in 2003 and 2005, respectively. 




Gerard J. M. Janssen (M'93) received the M.Sc.E.E. degree from Eindhoven University of Technology, Eindhoven, The Netherlands, in 1986 and the Ph.D. degree from Delft University of Technology (TUDelft), Delft, The Netherlands, in 1998.

In 1986, he joined the Physics and Electronics Laboratory, Dutch Organization of Applied Scientific Research (TNO) where he was involved in radar-cross-section modeling, radio direction finding, interference cancellation and wideband propagation measurements. He is currently an Associate Professor in the Wireless and Mobile Communications Group, Delft University of Technology. His research interests are in wireless communication, especially narrowband multiuser detection, digital modulation techniques, channel modelling, diversity techniques and ultrawideband communications and positioning.



Homayoun Nikookar (SM'03) received the Ph.D. degree in electrical engineering from Delft University of Technology (TUDelft), Delft, The Netherlands, in 1995.

$\mathrm{He}$ is an Associate Professor at the International Research Centre for Telecommunications and Radar (IRCTR) of the Department of Electrical Engineering, Mathematics and Computer Science, TUDelft. He is also the Coordinator of the Radio Advanced Technologies and Systems (RATS) Program at IRCTR. He has conducted research in many areas of wireless communications, including wireless channel modeling, UWB, MIMO, multicarrier transmission, wavelet-based OFDM and cognitive radio.

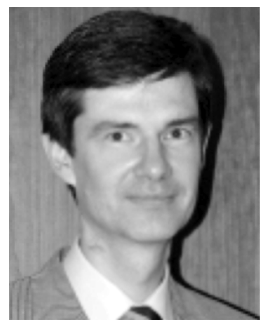

Alexander G. Yarovoy (M'96-SM'04) graduated with the Diploma (with honors) in radiophysics and electronics and received the Cand. Phys. Math. Sci. and Dr. Phys. Math. Sci. degrees in radiophysics all from Kharkov State University, Ukraine, in 1984, 1987, and 1994, respectively.

In 1987, he joined the Department of Radiophysics, Kharkov State University, as a Researcher and became a Professor in 1997. From September 1994 through 1996, he was with the Technical University of Ilmenau, Germany, as a Visiting
Researcher. Since 1999, he is with the International Research Centre for Telecommunications-Transmission and Radar (IRCTR), Delft University of Technology, The Netherlands, where he coordinates all UWB-related projects. His main research interests are in ultrawideband (UWB) technology and its applications (in particular, UWB radars) and applied electromagnetics (in particular, UWB antennas).

Prof. Yarovoy is the recipient of a 1996 International Union of Radio Science (URSI) "Young Scientists Award" and a co-recipient of the European Microwave Week Radar Award in 2001 for the Paper that Best Advances the State-of-the-Art in Radar Technology. He served as the Co-Chairman and the Technical Program Committee Chair of the Tenth International Conference on Ground Penetrating Radar (GPR2004), Delft, and the Secretary of the 1st European Radar Conference (EuRAD'04), Amsterdam, The Netherlands.

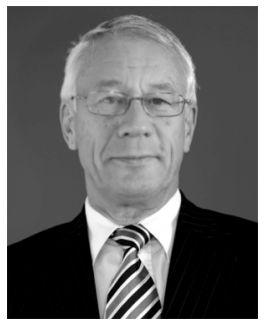

Leo P. Ligthart (F'02) was born in Rotterdam, The Netherlands, on September 15, 1946. He received the Engineer's degree (cum laude) and the Doctor of Technology degree from Delft University of Technology, Delft, The Netherlands, in 1969 and 1985, respectively, and the Doctorates (honoris causa) degree from Moscow State Technical University of Civil Aviation, Moscow, Russia, in 1999, and the Doctorates (honoris causa) degree from Tomsk State University of Control Systems and Radioelectronics, Tomsk, Russia, in 2001.

Since 1992, he has held the Chair of Microwave Transmission, Radar and Remote Sensing in the Department of Information Technology and Systems, Delft University of Technology. In 1994, he became Director of the International Research Center for Telecommunications-Transmission and Radar. His principal areas of specialization include antennas and propagation, radar and remote sensing, but he has also been active in satellite, mobile, and radio communications. 